\title{
Dynamic Rheological Properties of Polypropylene/Polyamide-6 Blends Modified with a Maleated Thermoplastic Elastomer
}

\author{
Hongzhi LIU, ${ }^{\dagger}$ Tingxiu XIE, Yuchun Ou ${ }^{\dagger}$ Xiaoping FAng, and Guisheng YANG ${ }^{\dagger}$ \\ Joint Laboratory of Polymer Science and Material, Institute of Chemistry, Chinese Academy of Sciences, \\ Beijing 100080, People's Republic of China
}

(Received April 21, 2004; Accepted June 15, 2004; Published September 15, 2004)

\begin{abstract}
The effects of added maleated thermoplastic elastomer (TPEg) on rheological properties and morphologies of polypropylene (PP)/polyamide-6 (PA6) blends are examined. For comparison, non-maleated version (TPE) is also added to the above binary blends. The magnitude of complex viscosity and viscoelastic moduli of PP/ PA6/TPE blends gradually decrease with the content of TPE. However, for PP/PA6/TPEg blends, these above parameters increase dramatically with increasing content of reactive TPEg and shear-thinning behavior becomes more obvious. SEM micrographs show that the addition of TPEg effectively increases compatibility between PP and PA6 and results in a considerable decrease in the domain size of dispersed phase; whereas the ternary blends containing nonreactive TPE exhibit a typical characteristic of immiscible blends with a very coarse morphology. TEM results further reveal that for PP/PA6/TPEg blends, a core-shell structure consisting of PA6 particles encapsulated with POE are probably formed in PP matrix. [DOI 10.1295/polymj.36.754]

KEY WORDS Polypropylene (PP) / Polyamide-6 / Rheological / Morphology / Interfacial / Adhesion /
\end{abstract}

In recent decades, polypropylene/polyamide (PP/ PA) blends have received much attention because they combine the insensitivity to humid environments and easy processing of the former with the thermomechanical characteristics of the latter. Unfortunately; they are incompatible polymer pairs because of their different polarities and crystalline morphology. Therefore, much effort has been particularly devoted to the compatibilization of these blends. Functionalized polypropylenes, such as maleic anhydride (MA) grafted PP, are effective compatibilizer for these blends. ${ }^{1-5}$ However, high levels of toughness can only be achieved by addition of an appropriate rubber that can function as an impact modifier. ${ }^{6-10}$ Many studies have demonstrated that maleated rubbers such as styrene-ethylene/butylene-styrene triblock copolymers (SEBS- $g$ MA) ${ }^{11-19}$ ethylene propylene random copolymer (EPR- $g$-MA) $)^{13-15}$ and poly(octene-ethylene) (POE- $g$ $\mathrm{MA})^{20}$ can effectively act as both impact modifier and compatibilizer for PP/PA6 blends.

We have developed a maleic anhydride grafted thermoplastic elastomer (TPEg) and successfully used it as an impact modifier of polyamide-6 (PA6) ${ }^{21,22}$ and the amorphous copolyester (PETG) ${ }^{23-25}$ This thermoplastic elastomer is a mixture of poly(octene-ethylene) (POE) and semicrystalline polyolefin. Compared with widely used maleated rubbers such as SEBS and EPR, it possesses the following advantages: lower cost, finer dispersion, better processability, and com- patibility with the PP matrix because of the incorporation of $40 \mathrm{wt} \%$ polyolefin plastics with a low melt viscosity into POE rubber.

In a previous work, TPEg was employed to compatibilize and toughen PP/PA6 blends. ${ }^{26}$ The addition of TPEg not only improved compatibility between PP matrix and PA6, resulting in a fine dispersion of PA6 in the PP matrix, but also significantly enhanced their fracture toughness in comparison to the PP/ PA6/TPE blends.

The flow properties of polymer blends depend, among other factors, on the morphology and on the interfacial properties. A modification of morphology by added compatibilizers, e.g., reduction in particle size, can result in a change in flow properties of the blends. ${ }^{27}$ As the rheological behavior of immiscible polymer blends is generally very complex, the effect of compatibilizer addition on rheological properties of such blends remained unclear in many aspects. ${ }^{28}$ In spite of some studies concerned with rheological properties of compatibilized PP/PA6 blends, ${ }^{4,29-33}$ there are limited papers dealt with their dynamic rheological behaviors, ${ }^{32,33}$ which is especially sensitive to the change in the morphology.

Therefore, the main purposes of this work is to investigate effects of addition of TPEg on dynamic rheological behaviors of PP/PA6 blends and make a further observation on their microstructures. For comparison, non-maleated thermoplastic elastomer, i.e.,

${ }^{\dagger}$ To whom correspondence should be addressed (Tel: 86-10-82618530, E-mail: hzliu@iccas.ac.cn). 
TPE, is also added to PP/PA6 blends, and rheological behaviors and morphologies of the blends prepared in the same condition are investigated.

\section{EXPERIMENTAL}

\section{Materials}

The PP used in this study was a commercial product (PP2401) from Yanshan Petrochemical Co., Ltd. with melt flow index $(\mathrm{MFI})=3.95 \mathrm{~g} / 10 \mathrm{~min}\left(230^{\circ} \mathrm{C}, 2.16\right.$ $\mathrm{kg})$. PA6 resin was supplied by Shanghai Plastics Production Factory No. 18 (Shanghai, China) with the relative viscosity equal to 2.6-2.9.

The interfacial modifier (TPEg) was a maleic anhydride grafted mixture of poly(octene-ethylene) (POE) rubber and a semicrystalline polyolefin plastic (60/40 by weight). Details about the functionalization procedure were reported elsewhere. ${ }^{22}$ Its grafting maleic anhydride content and MFI were $1 \mathrm{wt} \%$ and $4.11 \mathrm{~g} /$ $10 \mathrm{~min}\left(230^{\circ} \mathrm{C}, 2.16 \mathrm{~kg}\right)$, respectively. The POE used is a commercial product from Dupont DOW Elastomers (Wilmington, DE) under the trade name Engage 8445. Its octene content and MFI were 9.5\% and $3.5 \mathrm{~g} / 10 \mathrm{~min}$, respectively. The corresponding nongrafted mixture was also prepared and designated as TPE.

\section{Blend Preparation}

The blends were prepared by melt extrusion with a $\phi 30 \mathrm{~mm}$-twin-screw extruder with an L/D ratio of 23.2 (SHJ-30, Nanjing Plastic Machinery Co., Ltd., Nanjing, China) at $250 \mathrm{rpm}$. The content of PA-6 was fixed at $30 \mathrm{wt} \%$ in all the blends The barrel temperature was $220^{\circ} \mathrm{C}$. The blends were pelletized, dried, and injection-molded into standard tensile, flexural, and Izod impact test specimens with an injection-molding machine (SZ-160/80 NB, Ningbo Plastics Machinery Co., Ltd., Ningbo, Zhejiang, China) at $220^{\circ} \mathrm{C}$.

\section{Dynamic Rheological Measurement}

A parallel-plate rheometer (Rheometric Scientifics, DSR-200) was used to measure the dynamic viscosity $\left(\eta^{*}\right)$, storage modulus $\left(G^{\prime}\right)$, and loss modulus $\left(G^{\prime \prime}\right)$ of the blends at $230^{\circ} \mathrm{C}$ under nitrogen. The parallel plate used was $25 \mathrm{~mm}$ in diameter and the internal distance between the plates was $1.0 \mathrm{~mm}$.

\section{Morphological Observations}

The morphology of the blends was studied by transmission electron microscopy (TEM) using a Hitachi S-530 instrument, and by scanning electron microscopy (SEM) using a Hitachi-560 instrument. Scanning electron microscopy was used to study impact-fractured surfaces of the blend samples. The surfaces were coated with gold before observation to avoid charging under an electron beam.

The ultra thin samples for the TEM observation were obtained by microtoming molded specimen using an ultramicrotome and stained with ruthenium tetraoxide $\left(\mathrm{RuO}_{4}\right)$ vapor.

\section{RESULTS AND DISCUSSION}

\section{Dynamic Rheological Properties}

Figure 1a shows plots of complex viscosity $\left(\eta^{*}\right)$ as a function of frequency $(\omega)$ for PP/PA6/TPE blends with different weight percent of TPE. A progressive decrease in complex viscosity with the content of the TPE can be observed over the entire frequency. This may be due to the lower melt viscosity of TPE relative to PP matrix. It also noted that all blends exhibit a typical shear-thinning behavior.

The effect of TPEg addition on the complex viscosity of the PP/PA6 blends is presented in Figure $1 \mathrm{~b}$. When small amount of TPEg $(6 \mathrm{wt} \%)$ is added into the binary blend, the magnitude of complex viscosity
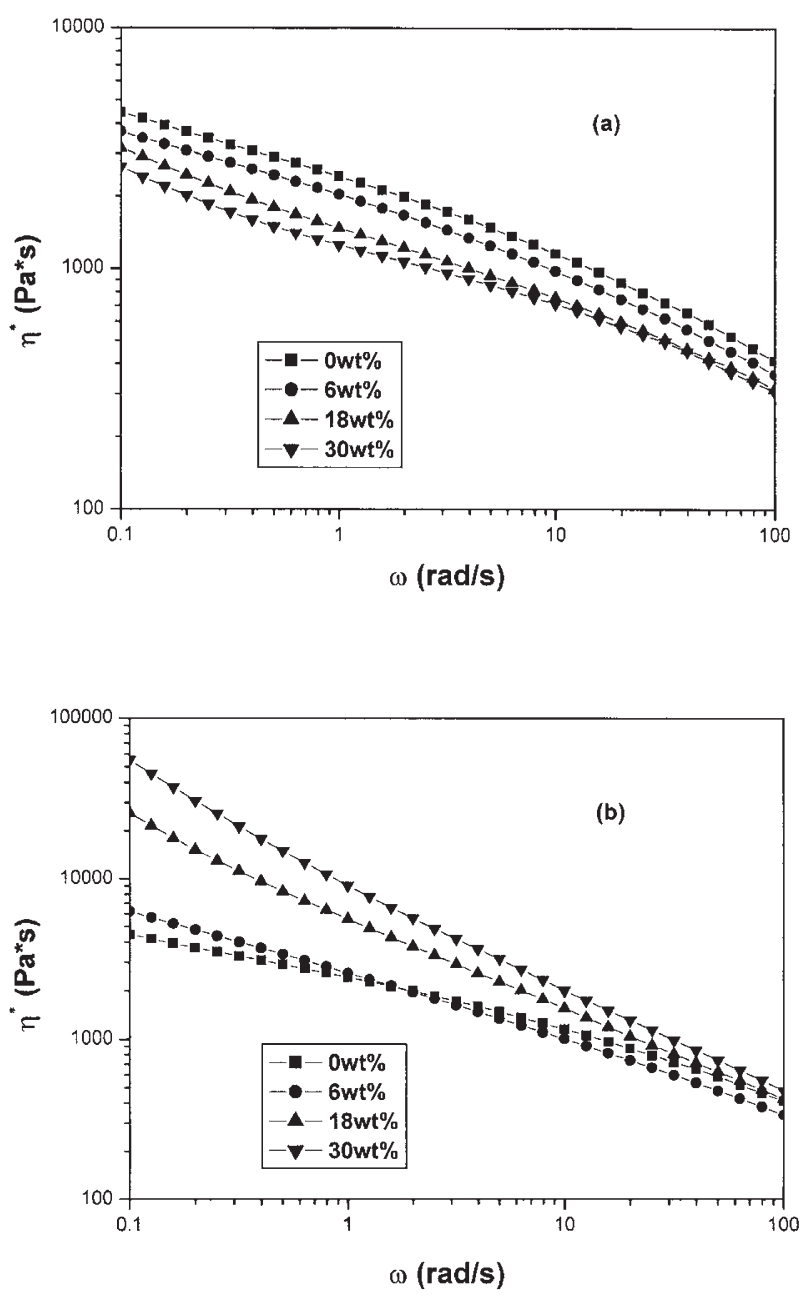

Figure 1. Plots of complex viscosity vs. frequency for: (a) PP/PA6/TPE; (b) PP/PA6/TPEg. 
presents an insignificant increase at low frequency and even the slight decrease at high frequency, compared with the pure PP/PA-6 blend. The situation is quite different for higher amount of TPEg blends. A significant increase in complex viscosity with further increasing of TPEg content is observed, the maximum increase being at low frequency. In addition, shearthinning behavior of these blends becomes more pronounced. Because the viscosity of TPEg is slightly lower than that of PP matrix as indicated from values of MFI, this significant enhancement of viscosity of the compatibilized blends can solely be attributed to the enhanced interfacial adhesion resulting from the chemical reaction of the anhydride group of TPEg with the terminal amino group of PA6 as shown below. ${ }^{21}$ The increase in viscosities was also observed in other compatibilized PP/PA6 blend systems. ${ }^{4,29}$

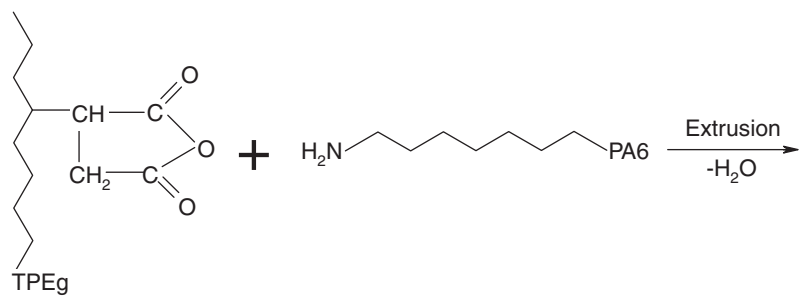

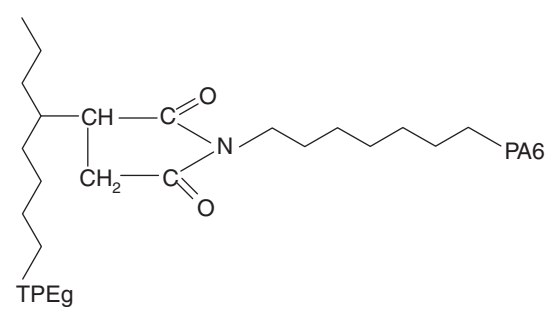

The processing pressure can give an indication of viscosity during the compounding process, which arises from chemical reaction and/or physico-chemical interaction. Figure 2 shows the variation of processing pressure of the PP/PA6/TPEg and PP/PA6/TPE

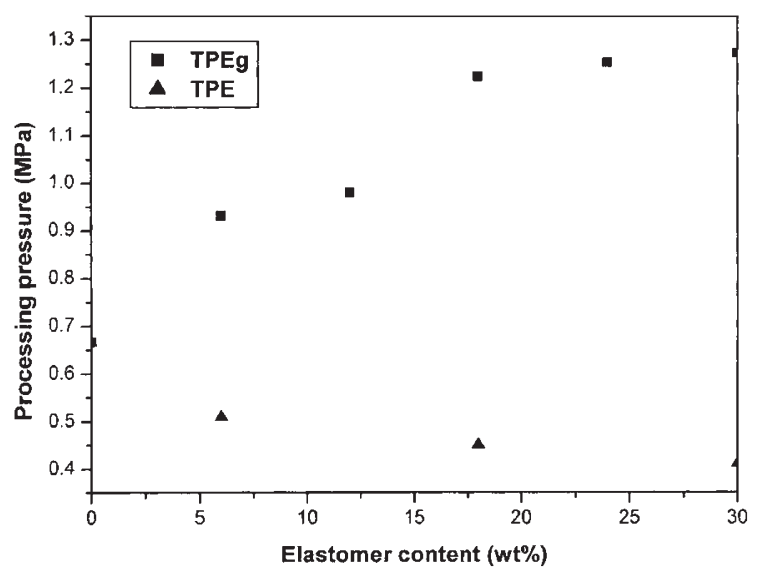

Figure 2. Variations of processing pressure vs. weight content of both thermoplastic elastomers. blends with weight content of the elastomer. As the amount of TPEg increases, processing pressure increases rapidly, while for PP/PA6/TPE blends, an opposite trend occurs. The result is in good accordance with that of complex viscosity (Figure 1).

The dynamic storage modulus $\left(G^{\prime}\right)$ is related to the elastic behavior of the material and may be considered as the storage energy. The dynamic loss modulus $\left(G^{\prime \prime}\right)$ represents the dissipated energy. The dependence of $G^{\prime}$ and $G^{\prime \prime}$ on the frequency measures the relative motion of all molecules in the bulk and can give important information about the flow behavior of melts.

Figures 3 and 4 show dependences of viscoelastic moduli $\left(G^{\prime}\right.$ and $\left.G^{\prime \prime}\right)$ on frequency for PP/PA6/TPEg and PP/PA6/TPE blends, respectively. It is observed that both $G^{\prime}(\omega)$ and $G^{\prime \prime}(\omega)$ of PP/PA6/TPEg blends are always higher than those of the corresponding PP/PA6/TPE blends. The higher viscoelastic moduli of the former may attribute to the enhanced interaction between phases caused by compatibilization of TPEg, which reduces the possibility of the interlayer slip, and increases formation of associative network
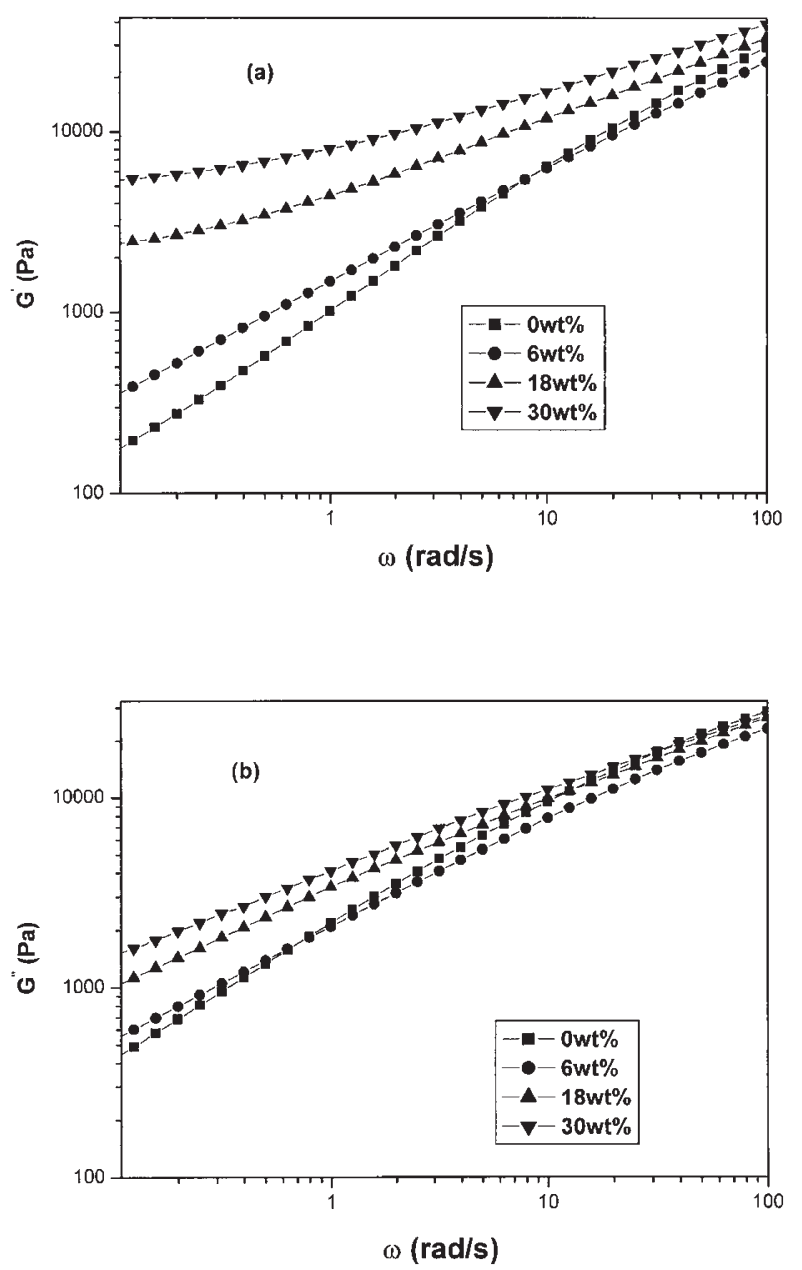

Figure 3. Plots of viscoelastic moduli vs. frequency for PP/ PA6/TPEg blends: (a) storage modulus; (b) loss modulus. 

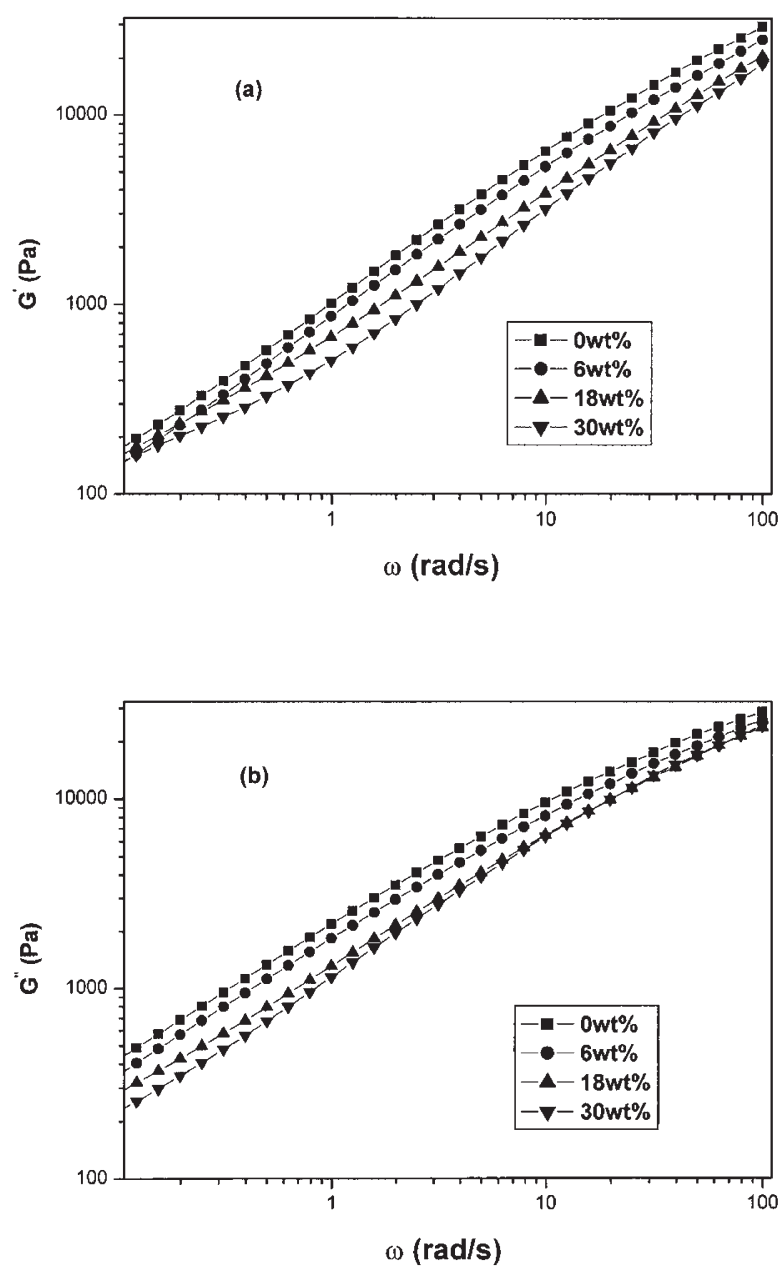

Figure 4. Plots of viscoelastic moduli vs. frequency for PP/ PA6/TPE blends: (a) storage modulus; (b) loss modulus.

formation between the interfaces. The effect of TPEg percentage on the viscoelastic moduli for PP/PA6/ TPEg blends is similar to that on complex viscosity. When the added TPEg percentage is low $(6 \mathrm{wt} \%)$, the values of moduli are higher than those of pure PP/PA6 blend at low frequency, whereas at high frequency the moduli are slightly lower instead. When the content of TPEg further increases, the enhancement tendency becomes very obvious, especially at low frequency and the increasing degree is larger for storage modulus. However, for PP/PA6/TPE blends, the storage modulus and loss modulus decrease gradually with the content of TPE in the blends (Figure 4). The significant difference in the rheological behaviors between above two ternary blend systems accompanies the different morphologies, as indicated by the micrographs in the following section.

\section{Morphological Observation}

SEM micrographs of impact-fractured surfaces of $\mathrm{PP} / \mathrm{PA} 6 / \mathrm{TPE}$ blends containing varying weight percent of TPE are presented in Figure 5. Among them, the uncompatibilized PP/PA6 blend (Figure 5a) ex- hibits a very coarse and large morphology, consisting of spherical PA6 particles with size-scales of between 7 and $10 \mu \mathrm{m}$ in diameter in the PP matrix. The poor adhesion between two phases is also evident from micrograph of fracture surface, with particles of PA6 pulled from the smooth holes lying loose on the fracture surface.

After addition of TPE into the above binary blend, the blend domains become more irregular, as indicated in Figure $5 b-d$ (note the difference in magnification of the three latter photographs with the former). However, the large and coarse morphologies still suggest characteristics of immiscible blends. Changes in morphology of PP/PA6 blends with addition of TPEg are observed in Figure 6. The particle size of the dispersed phase in PP/PA6/TPEg blends is decreased significantly with increasing amount of TPEg in the blends. When only $6 \mathrm{wt} \% \mathrm{TPEg}$ is added the average domain size decreases to about $2 \mu \mathrm{m}$ in diameter (Figure 6a), compared with the uncompatibilized PP/PA6 blend (Figure 5a). As the amount of TPEg increase further, the domain size continuously reduces to only $0.1-0.2 \mu \mathrm{m}$ (Figure 6b). Finally, one cannot almost identify the difference between the dispersed and matrix phase at the present magnification (Figure 6c). This indicates that the added TPEg acts as an effective compatibilizer, which reduces the interfacial tension between PP matrix and PA6, and hence leads to a fine dispersion of PA6 particles in the matrix.

However, as it is impossible to clearly image the sub-micron dispersed phase morphologies using SEM, TEM was subsequently employed. As a representative example, Figure 7 shows the $\mathrm{RuO}_{4}$-stained TEM photographs of PP/PA6 blend modified with $18 \mathrm{wt} \%$ TPEg. In Figure $7 \mathrm{a}$ and $\mathrm{b}$, the dark gray and relative large areas represent PA6 domains (0.1$0.2 \mu \mathrm{m}$ ) dispersed in the PP matrix, which still remains unstained. Although $\mathrm{RuO}_{4}$ staining does not give a clear contrast between PA6 and TPEg domains in the ternary blend system, one can be still noted that PA6 particles seem to be encapsulated by a grayish interlayer to form a core-shell type structure. This morphology results from reaction between the maleic-anhydride grafts on the TPEg and the terminal amine groups of the PA6. As reported previously by Rösch, ${ }^{34}$ imide-coupling of TPEg with PA6 interface and surface tension gradients between PP and PA6 are responsible for the accumulation of the TPEg at the surface of PA6 inclusions, which results in modified particles with single PA6 core encapsulated in an ultra thin POE-shell. The interfacial layer acts as the dispersing agent, promoting PA6 dispersion in the PP matrix. 

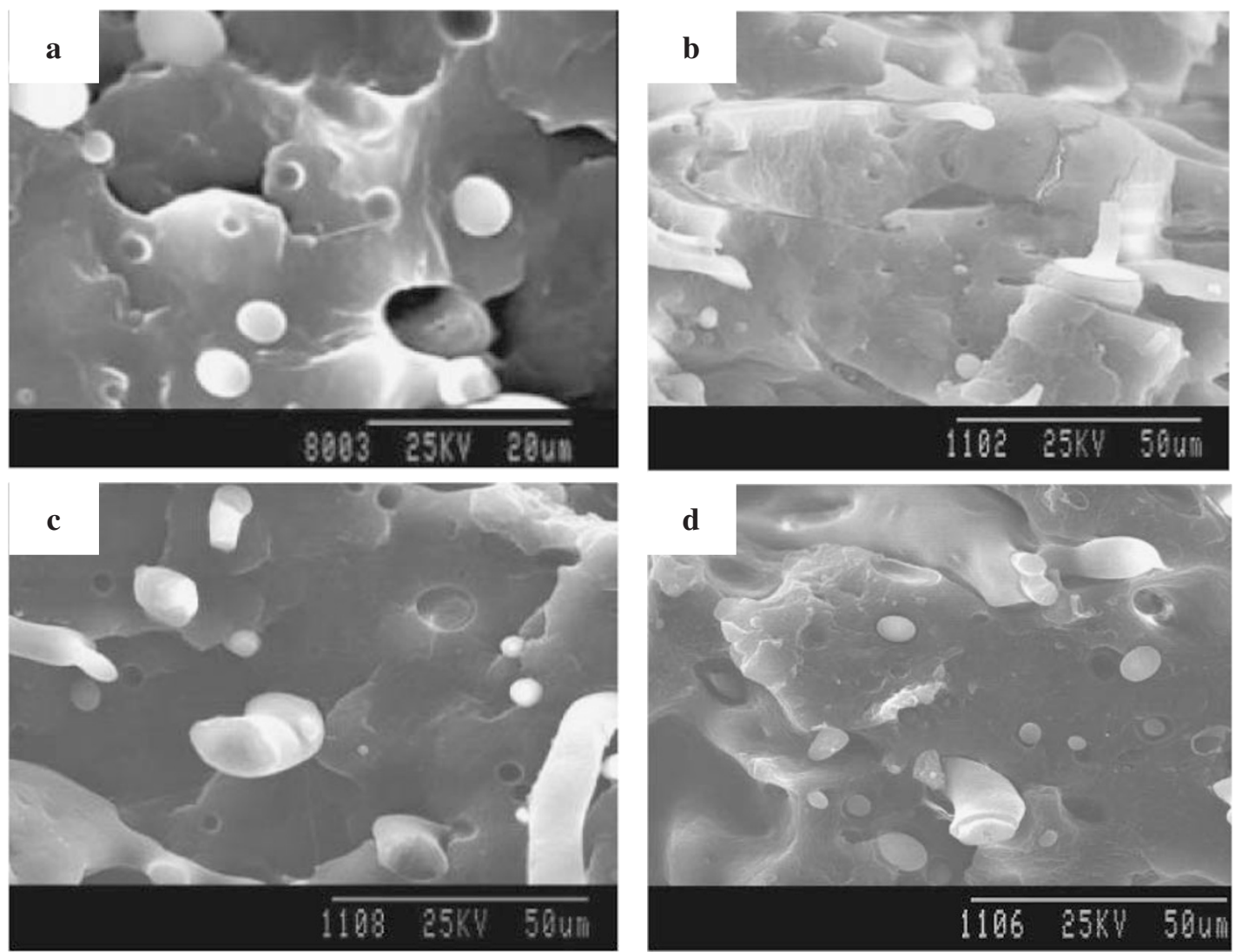

Figure 5. SEM micrographs of fractured surfaces of PP/PA6/TPE with varying weight percent of TPE: (a) 70/30/0; (b) 64/30/6; (c) $52 / 30 / 18$; (d) $40 / 30 / 30$.
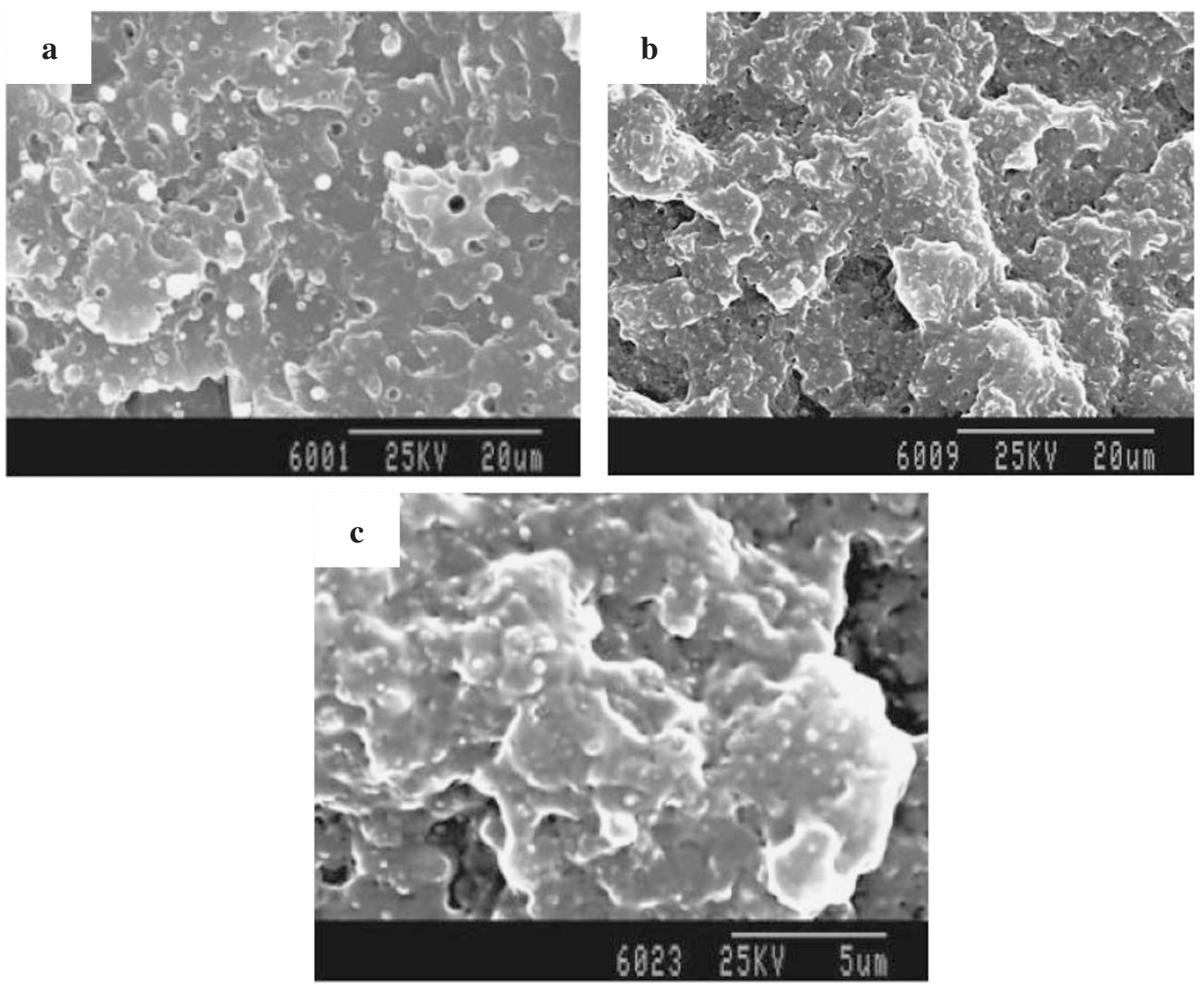

Figure 6. SEM micrographs of fractured surfaces of PP/PA6/TPEg with varying weight percent of TPEg: (a) 64/30/6; (b) 52/30/18; (c) $40 / 30 / 30$. 

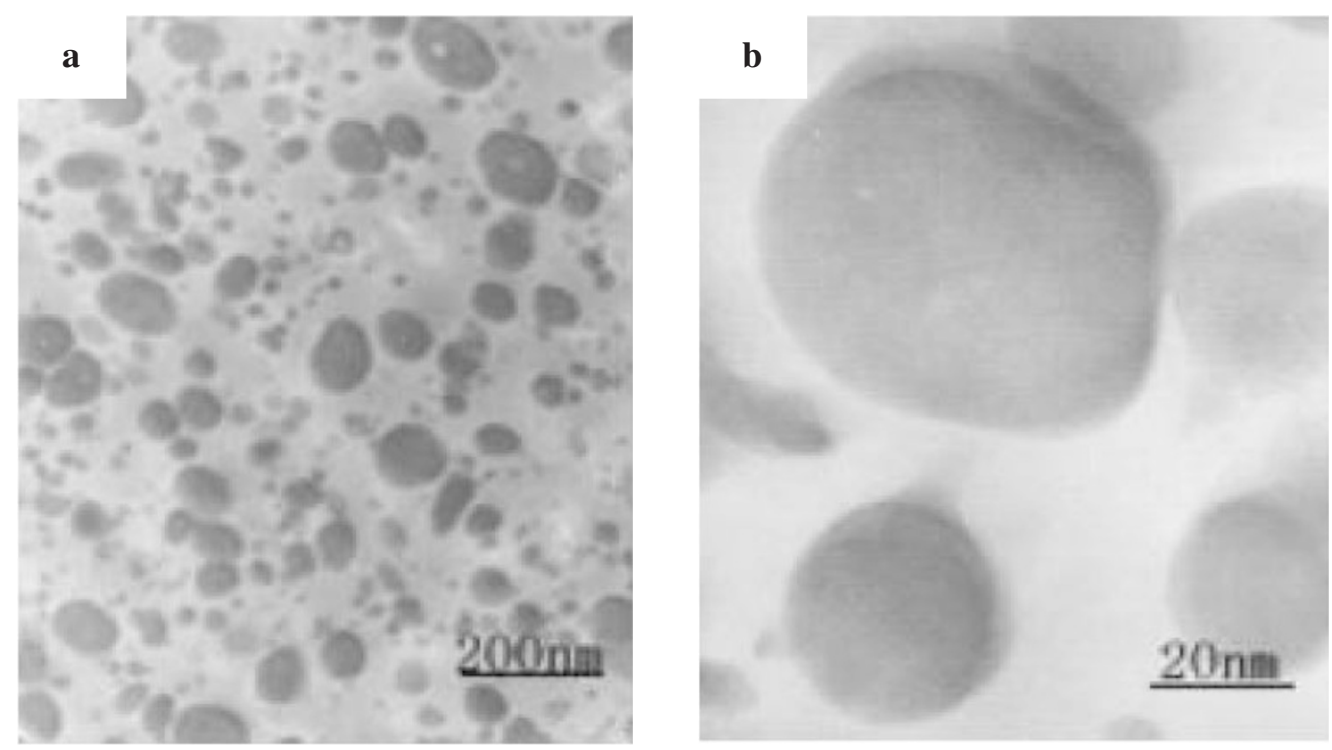

Figure 7. TEM micrographs of the $\mathrm{RuO}_{4}$-stained PP/PA6 blend modified with 18 wt \% TPEg: (a) low magnification; (b) high magnification.

\section{CONCLUSIONS}

In this study, the dynamic rheological properties and microstructures of PP/PA6/TPEg blends were investigated, together with those of $\mathrm{PP} / \mathrm{PA} 6 / \mathrm{TPE}$ blends. Two ternary blend systems exhibit quite different dynamic rheological behaviors. For PP/PA6/ TPE blends, the complex viscosity and viscoelastic moduli progressively decrease with increasing TPE content. On the other hand, addition of TPEg dramatically increases the complex viscosity and moduli when TPEg content is higher. This is due to enhanced interfacial adhesion, which is caused by in-situ compatibilization of TPEg. More specifically, short-range effects such as a frictional force in the surrounding medium, and restriction from the new adjacent repeating unit of the same molecule including TPEg play an important role. Additionally shear-thinning behaviors also become more evident for PP/PA6/TPEg blends. The significant difference in rheological behaviors between them accompanies the different morphologies, which result from compatibilizing role of TPEg. Although addition of TPE makes morphologies of PP/ PA6 binary blend more complex, PP/PA6/TPE blends still demonstrate typical immiscible characteristics with coarse and large domains, which further indicates the poor adhesion between the PP matrix and dispersed PA6 particles. However, when TPEg is added, the size of the dispersed PA6 phase significant decreases with increasing the amount of TPEg in the blends. Furthermore, from TEM micrographs of PP/ PA6/TPEg blends, it seems that core/shell particles consisting of PA6 nanoparticle core and POE shell are formed in the PP matrix.
Acknowledgment. The authors are grateful to the National Natural Science Key Fundation of China for financial support (Contract No. 10032010).

\section{REFERENCES}

1. F. Ide and A. Hasegawa, J. Appl. Polym. Sci., 18, 963 (1974).

2. T. Nishio, Y. Sukuki, K. Kojima, and M. Kakugo, "Proceedings of the IUPAC International Symposium on Speiciality Polymers," Hütig and Wepf, Basel, 1991, p 51.

3. S. S. Dagli, M. Xanthos, and J. A. Biesenberger, Soc. Plast. Eng., ANTEC, 36, 1924 (1990).

4. R. M. Hölsti-Miettinen and J. Seppälä, Polym. Eng. Sci., 32, 868 (1992).

5. A. González-Montiel, H. Keskkula, and D. R. Paul, J. Polym. Sci., Part B: Polym. Phys., 33, 1751 (1995).

6. R. Gelles, R. G. Lutz, and W. P. Gergen, Eur. Patent Appl. 0261748 (1987).

7. T. Nishio, T. Yokoi, T. Nomura, K. Ueno, T. Akagawa, I. Sakai, and Y. Takaski, U.S. Patent 4988764 (1990).

8. M. J. Modic and L. A. Pottick, Plast. Eng., 47, 37 (1991).

9. M. J. Modic and L. A. Pottick, Soc. Plast. Eng., ANTEC, 37, 1907 (1991).

10. M. J. Modic, Soc. Plast. Eng., ANTEC, 39, 205 (1993).

11. A. N. Wilkin, L. Laugel, M. L Clemens, V. M. Harding, and M. Marin, Polymer, 40, 4971 (1998).

12. J. Rösch and R. Mülhaupt, Makromol. Chem., Rapid Commun., 14, 503 (1993).

13. J. Rösch, Polym. Eng. Sci., 35, 1917 (1995).

14. J. Rösch and R. Mülhaupt, Polym. Bull. 32, 697 (1994).

15. J. Rösch, R. Mülhaupt, and G. H. Michler, Macromol. Symp. 112, 141 (1996).

16. G.-M. Kin, G. H. Michler, J. Rösch, and R. Mülhaupt, Acta Polym., 49, 88 (1998).

17. M. Tasdemir, J. Appl. Polym. Sci., 89, 3485 (2003). 
18. B. Ohlsson, H. Hassander, and B. Törnell, Polymer, 39, 4715 (1998).

19. B. Ohlsson, H. Hassander, and B. Törnell, Polymer, 39, 6705 (1998).

20. N. Zeng, S.-L. Bai, C. G. Sell, J.-M. Hiver, and Y.-W. Mai, Polym. Int., 51, 1439 (2002).

21. Z.-Z. Yu, M. Lei, Y.-C. Ou, and G.-H. Hu, J. Polym. Sci., Part B: Polym. Phys., 37, 2664 (1999).

22. Z.-Z. Yu, Y.-C. Ou, Z.-N. Qi, and G.-H. Hu, J. Polym. Sci., Part B: Polym. Phys., 36, 1987 (1998).

23. Z.-Z Yu, M. Lei, Y.-C. Ou, and G.-S. Yang, Polymer, 43, 6993 (2002).

24. Z.-Z. Yu, M. Lei, Y.-C. Ou, G.-S. Yang, and G.-H. Hu, J. Polym. Sci., Part B: Polym. Phys., 38, 2801 (1999).

25. M. Lei, J.-F. Zhou, Z.-Z. Yu, Y.-C. Ou, and G.-S. Yang, Acta Polym. Sin., 5, 608 (2000).

26. Y.-C. Ou, Y.-G. Lei, X.-P. Fang, and G.-S. Yang, J. Appl. Polym. Sci., 91, 1806 (2004).
27. H. F. Guo, S. Packirisamy, R. S. Mani, C. L. Aronson, N. V. Gvozdic, and D. J. Meier, Polymer, 39, 2495 (1998).

28. L. A. Utracki and M. R. Kamal, in "Polymer Blends Handbook," L. A. Utracki, Ed., Kluwer Academic, Dordrecht, 2003, chapt. 7, p 517.

29. C. Marco, G. Ellis, M. A. Gómez, J. G. Fatou, J. M. Arribas, I. Canpoy, and A. Fontecha, J. Appl. Polym Sci., 65, 2665 (1997).

30. S. J. Park, B. K. Kim, and H. M. Jeong, Eur. Polym. Sci., 26, 131 (1990).

31. B. Ohlsson, H. Hassander, and B. Törnell, Polymer, 39, 6706 (1998).

32. D. Shi, Z. KE, J.-H. Yang, Y. Gao, J. Gao, J. Wu, and J.-H. Yin, Macromolecules, 35, 8005 (2002).

33. S. Schlag, J. Rösch, and Chr. Freidrich, Polym. Bull., 30, 603 (1993).

34. J. Rösch and R. Mülhaupt, J. Appl. Polym. Sci., 56, 1599 (1995). 